\title{
Actuator and Process Development for Vibration Assisted Turning of Steel
}

\author{
Martin SCHWARZE ${ }^{\mathrm{a}, 1}$, Carlo RÜGER ${ }^{\mathrm{a}}$, Oliver GEORGI ${ }^{\mathrm{a}}$, Hendrik RENTZSCH ${ }^{\mathrm{a}}$ and \\ Holger PÄTZOLD ${ }^{\mathrm{b}}$ \\ ${ }^{a}$ Fraunhofer Institute for Machine Tools and Forming Technology IWU, Chemnitz, \\ 09126 Germany \\ ${ }^{\mathrm{b}}$ Schaeffler Technologies AG \& Co KG, Herzogenaurach, 91074 Germany
}

\begin{abstract}
Due to continuous tool engagement, turning processes tend to form long chips when machining ductile materials. These chip shapes have a negative influence on process performance and productivity. One approach to improve chip breakage is superimposition of vibrations in feed direction of the turning process, which leads to a modulation of uncut chip thickness. In a joint industrial project with Schaeffler Technologies AG \& Co. KG, Fraunhofer IWU developed an oscillating actuator for turning. The actuator converts a rotational movement of a drive motor into a translational vibration via an eccentric gear. The tool shank is mounted in solid joint assemblies. With this prototypical system, a cyclic movement of the tool in feed direction can be realized. The typical operating parameters of the actuator is within the range of $1 \ldots 100 \mathrm{~Hz}$ with adjustable vibration amplitudes up to $0.6 \mathrm{~mm}$ peak-to-peak. A significant improvement in chip breaking during the machining of steel 1.0503 was shown in cutting tests.
\end{abstract}

Keywords. Manufacturing research into Industry, Sustainable Manufacturing, VAT, chip breaking.

\section{Introduction}

Turning is a cutting operation with defined cutting edge. Typical for this process is the continuous engagement that often leads to poor chip breaking. The formation of ribbon, tangled and flat spiral chips can evoke considerable problems in the chip flow. Especially during internal machining, this can damage the work piece or lead to tool breakage. Unfavourable chip shapes also tend to form chip nests, which inhibits automatic chip removal by the chip conveyor. Collision geometries occur in the working area and the accumulated heat of the nest can negatively influence the machine geometry. Therefore, a goal in turning is to generate short broken chips, independent of tool wear and batch variations for increased operational efficiency and chip flow resilience. This paper describes the approach of uncut chip thickness modulation, the developed actuator and presents the achieved chip shapes.

\footnotetext{
${ }^{1}$ Corresponding Author. +49 3715397 1266; e-mail: martin.schwarze@iwu.fraunhofer.de

${ }^{2}$ Corresponding Author. phone: +49 913282 86887; e-mail: paetzhlg@schaeffler.com
} 


\section{Kinematics of vibration assisted turning in feed direction}

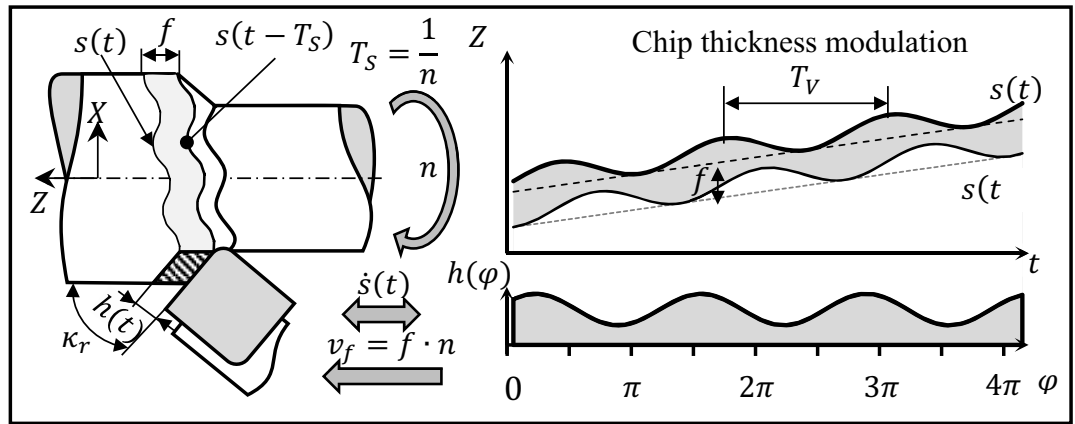

Figure 1. Kinematic VAT longitudinal cut (left) and chip thickness modulation (right)

The variation of the uncut chip thickness is a known principle to improve chip breaking [1]. For longitudinal cuts, an unidirectional oscillating motion $s(t)$ in feed direction is useful. During the manufacturing process, the feed velocity of the tool $v_{f}$ is superimposed with the oscillating speed $\dot{s}(t)$ of the tool and thus the feed is modulated (Figure 1). The uncut chip thickness $h(t)$ results from the projection of feed $f$ onto the tool cutting plane by the tool setting angle $\kappa_{r}$. On the right side, Figure 1 shows the unrolled tool paths and the uncut chip thickness function over the angle of rotation $\varphi$. The number of extreme values of $h(t)$ depends on the ratio of the periods $T_{V}$ and $T_{S}$. This ratio also defines the modulation factor $K$, which is the number of maxima during one spindle revolution. The phase shift between oscillation frequency and rotational speed which results due to the factor has a significant influence on the engagement of the cutting edge and the chip thickness modulation.

\section{Actuator overview}

Due to the advantages of vibration assisted turning, several vibration systems exist for processes with long continuous cutting engagement such as turning and drilling. The first publications on vibration assisted turning date back to the 1950's and 1960's [2]. Here, tools were excited with vibration generators in different tool axes. In the following, various vibration systems for low-frequency vibration assisted turning (LVAT) will be presented.

Sources [3] and [4] show hydraulic oscillating systems. With these systems, high cutting values could be achieved, but the amplitude was frequency-dependent. Other systems are driven by piezo actuators. Here [5] shows a piezoelectric boring bar configuration for turning and centric drilling in lathes. A piezoelectric tool was also presented in [6]. Here, the turning tool is equipped with a parallel spring mechanism made of solid-state joints. Both systems were tested in industrial use and improved chip breaking was demonstrated for low feed rates. However, the systems only achieve low amplitudes due to the characteristics of the piezo-actuators.

Table 1 shows vibration systems with different drive principles with the associated process parameters and vibration characteristics of chip breaking tests. The solutions listed are limited to direct actuation of the tool in the feed direction. 
Table 1. Vibration characteristics and process parameters of actuator systems

\begin{tabular}{cccccccc}
\hline \multirow{2}{*}{ System } & \multicolumn{2}{c}{ Vibration characteristics } & & \multicolumn{4}{c}{ Process parameters } \\
\cline { 2 - 3 } & $\begin{array}{c}\text { Amplitude } \hat{A} \\
{[\mathrm{~mm}]}\end{array}$ & $\begin{array}{c}\text { Frequency } f_{V} \\
{[\mathrm{~Hz}]}\end{array}$ & & $\begin{array}{c}\text { Feed } f \\
{[\mathrm{~mm}]}\end{array}$ & $\begin{array}{c}\text { Cutting deth } a_{p} \\
{[\mathrm{~mm}]}\end{array}$ & $\begin{array}{c}\text { Cutting speed } v_{c} \\
{[\mathrm{~m} / \mathrm{min}]}\end{array}$ & Material \\
\hline Electrohydraulic [3] & 0.1 & 125 & & 0.91 & 0.76 & 137 & Mild Steel \\
Electrohydraulic [4] & 0.15 & 30 & & 0.4 & n.a. & n.a. & C45E \\
Piezoelectric [5] & 0.11 & 20 & & 0.1 & 0.5 & 226 & SHP 440 \\
Piezoelectric [6] & 0.05 & 25 & & 0.05 & 1 & 550 & CGI \\
\hline
\end{tabular}

\section{Design of actuator for VAT}

\subsection{Kinematic structure and assembly of the actuator}

The actuator is designed for internal machining in wheel bearing production. Figure 2 shows the technical principle and the assembly of the Schaeffler actuator. Here, a boring bar (1) is supported via two packages of solid-state joints (2). The solid-state joints are designed as membranes and are clamped against each other via a sleeve (3). Any bending moments that occur are thus absorbed by both bearing points. The solid-state joints allow for boring bar movement without play and friction. The torsional moment of the boring bar is absorbed by a shaft-hub connection in the first bearing point. The boring bar is exchangeable or has an exchangeable head interface (4).

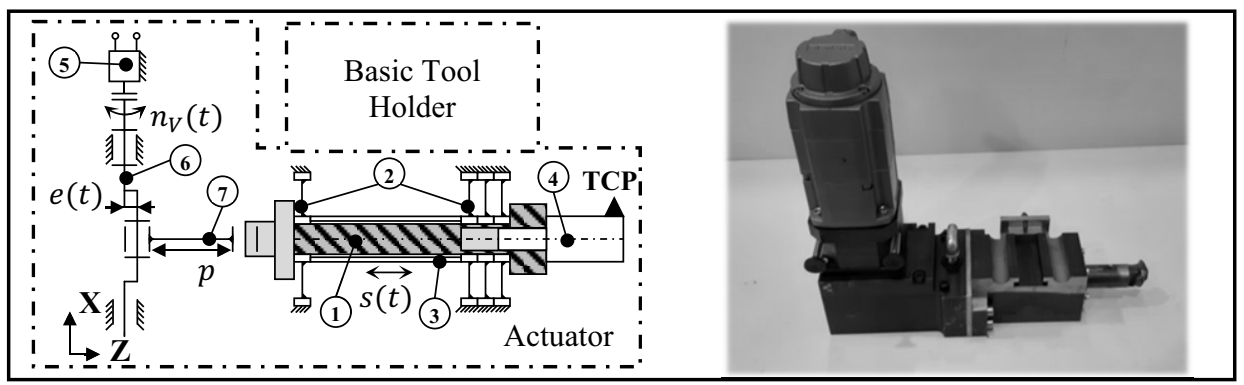

Figure 2 Kinematics of the actuator (left) and Schaeffler VAT-actuator (right)

During development, the solid-state joint membranes were first optimised using the FE method to achieve a balanced stress distribution with high radial stiffness and axial rigidity. During the design, load regimes were determined using the technology data of vibration assisted turning and the stress distribution was optimised by the design of the membrane thickness, the diameter ratios and the radii. Subsequently, the optimal arrangement of the solid-state joints within a standard change holder was determined via a iterative parameter study.

The oscillating movement $s(t)$ is generated via a thrust crank mechanism (Figure 3 left). A NC servo motor (5) drives a crankshaft with a speed $n_{V}$ of up to $6000 \mathrm{rpm}$. The shaft contains a double eccentric mechanism (6) which allows the eccentricity and thus amplitude $A$ between $0.02 \mathrm{~mm}$ and $0.3 \mathrm{~mm}$. A connecting rod (7) with length of $p$ connects the crank to the boring bar. The transmission function of the mechanism results in equation 2. of the oscillating movement $s(t)$. 


$$
s(t)=A \cdot \cos \left(2 \pi n_{V} \cdot t\right)+p \cdot \sqrt{1-\frac{A^{2} \cdot \sin ^{2}\left(2 \pi n_{V} \cdot t\right)}{p^{2}}}
$$

The serial arrangement is characterised by the fact that only low bending moments are induced from the drive train into the boring bar bearing and in addition, the forced guidance allows pulling cuts to be made.

\subsection{Machine integration of actuator}

After testing a prototype, it was possible to implement an actuator for industrial use in wheel bearing production with the same mode of operation (Figure 3). A combined PLC/NC-control concept was implemented to automatically select the optimum vibration speed $n_{V}(t)$ by multiplication with the modulation factor $K$ and the spindle speed $n(t)$. The acceleration and deceleration values of the actuator allow the calculation of the deceleration path $\Delta Z$. With the consideration of the current feed rate and position $Z(t)$ an automatic switching of the actuator in longitudinal cutting was implemented.

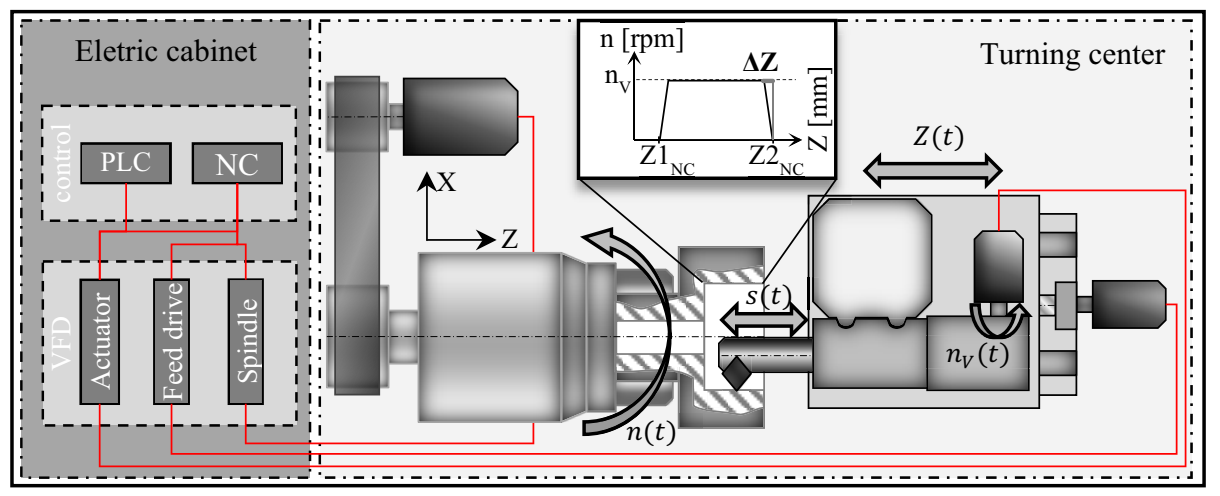

Figure 3 Actuator integration Schaeffler production plant

\section{Experimental investigation}

With the developed actuator cutting tests were executed. The focus was set on the influence of vibration excitation, especially the interaction of vibration frequency and amplitude and also the phase shift, on the chip shape and breaking behaviour.

\subsection{Experimental setup and investigation parameters}

The cutting tests were done on a NILES N20 CNC-Lathe. The work piece material was steel C45 (1.0503) as round bar, which was clamped in a three-jaw chuck. The investigated process was internal longitudinal turning with a starting diameter of $100 \mathrm{~mm}$. The tool was a Kennametal A32S DDPNR 15 boring bar with coated tungsten carbide grade P20 cutting inserts DNMG 150616. Inner coolant supply with 8\% oil emulsion with pressure 6 bar was applied to the cutting tool. Table 2 shows the experimental parameters. 
Table 2. Parameters for cuttings tests

\begin{tabular}{ccccc}
\hline & \multicolumn{2}{c}{ Cutting parameters } & \multicolumn{2}{c}{ Vibration parameters } \\
\hline $\begin{array}{c}\text { Cutting speed } v_{c} \\
{[\mathrm{~m} / \mathrm{min}]}\end{array}$ & $\begin{array}{c}\text { Feed } f \\
{[\mathrm{~mm}]}\end{array}$ & $\begin{array}{c}\text { Cutting depth } a_{p} \\
{[\mathrm{~mm}]}\end{array}$ & $\begin{array}{c}\text { Frequency } f_{V} \\
{[\mathrm{~Hz}]}\end{array}$ & $\begin{array}{c}\text { Amplitude Â (peak-peak) } \\
{[\mathrm{mm}]}\end{array}$ \\
\hline 190 & 0.1 & 0.5 & $13 \ldots 65$ & $0.08 \ldots 0.11$ \\
\hline
\end{tabular}

\subsection{Results}

The conventional base of operations were long conical helical chips with high snarling tendency like shown on left side in Figure 4, symbolized by two red parallel lines.

Applying VAT the chip shape was changed significantly. It was found that in intermediate cutting conditions, symbolized by sinusoidal crossing red lines, a single chip forms for each vibration cycle. With increasing frequency $f_{\mathrm{V}}$ the number of vibration cycles per rotation rises. As a result the number of chips also increases and the length of the single chip decreases. Like shown in Figure 4 not only the chip length, but also the shape of the chips changed with the vibration frequency. With an applied frequency of $13 \mathrm{~Hz}$, meaning $\mathrm{K}=1.5$ vibrations per rotation, short conical helical chips are formed. An increased frequency of $65 \mathrm{~Hz}(\mathrm{~K}=7.5)$ tended to form loose arc shaped chips.

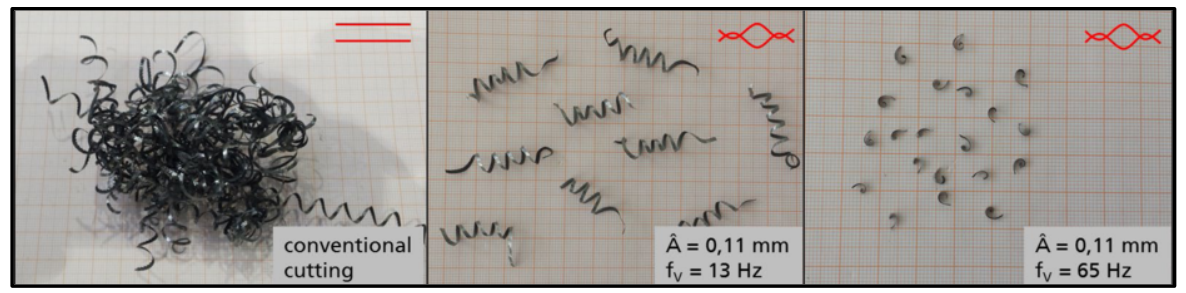

Figure 4 Chips formed with different vibration frequencies $\left(v_{c}=190 \mathrm{~m} / \mathrm{min}, \mathrm{f}=0.1 \mathrm{~mm}\right)$

The vibration amplitude affects the variation of uncut chip thickness. For intermediate cutting conditions, an amplitude $\hat{\mathrm{A}}$ (peak-peak) higher than the feed $\mathrm{f}$ is necessary. An amplitude below the feed causes continuous cutting conditions with a modulation of the uncut chip thickness. Hence the forming chip contains cyclic weak flaws which act as predetermined breaking points. With a certain probability the material fails and the chip breaks in this area. Because the chip breaking is not ensured for each single flaw the forming chip can contain several of them until it finally breaks. Chips generated with continuous cutting, symbolized by two not crossing sinusoidal red lines with $180^{\circ}$ phase shift, are shown in Figure 5. In this example the chips formed during the machining tests tended to break at the second flaw. Only in few cases chips broke after the first oscillation period. However, this behaviour is assumed to be stochastic and strongly dependent on the machined material properties and the process conditions. Because the chip breaks only with a certain probability on each flaw, the resulting chip lengths is on average longer than with interrupted cutting conditions on the same vibration frequency. Increasing the frequency leads to a shortening of the distance between and thus a higher number of flaws. More weak points are created in the cut off material. Even with increased vibration frequency the chips tend to break on the first or second flaw. This leads to a shortening of the resulting chip lengths. The cutting tests show that vibration assistance in continuous cutting conditions can also induce a considerable shortening and a targeted control of the resulting chip lengths. Despite the vibration superimposition the modulation of uncut chip thickness is almost neutralized 
with phase shift of $0^{\circ}$. Shown in Figure 5 (right), long conical helical chips, comparable with the chip shape of conventional cutting, occur.

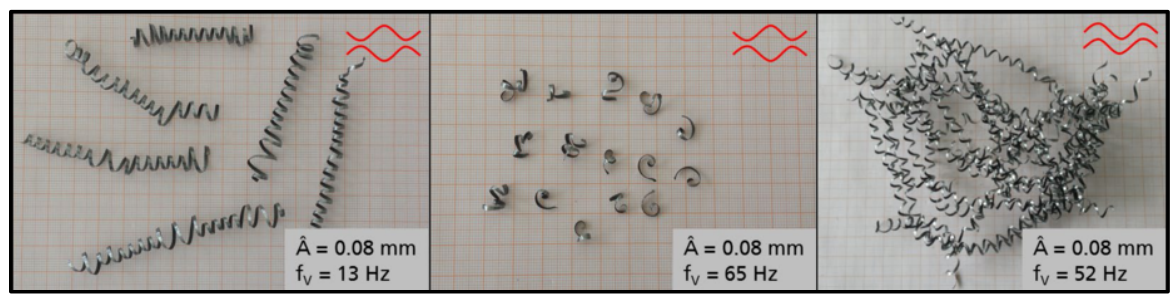

Figure 5 Chips formed with different vibration conditions $\left(\mathrm{v}_{\mathrm{c}}=190 \mathrm{~m} / \mathrm{min}, \mathrm{f}=0.1 \mathrm{~mm}\right)$

\section{Conclusion}

A new actuator for low frequency VAT was developed. The unit is capable for finishing and roughing operations with high vibrations amplitudes. With this actuator cutting tests were done on internal longitudinal turning. Especially the influence of vibration frequency, vibration amplitude and phase shift on the forming chips were investigated. The results show, that with VAT the chip breaking can be improved significantly. With the ratio of oscillation frequency and spindle speed, not only the chip length can be specifically adjusted, but also the chip shape can be influenced. It could be shown, that with increasing modulation factor $\mathrm{K}$ the chip length decreases. The experiments also indicate, that low frequency VAT in intermediate cutting conditions as well as in continuous cutting conditions lead to a significant improved chip breakage.

The system has been tested on a series machine under production conditions for wheel bearing components since development was completed. Controlled chip breaking increases machine availability by up to $5 \%$.

\section{Acknowledgements}

The actuator system and the machining process were developed and tested within an industrial research project with Schaeffler Technologies AG \& Co KG.

\section{References}

[1] A. Miyake, A. Kitakaze, S. Katoh, M. Muramatsu, K. Noguchi, K. Sannomiya, T. Nakaya, H. Sasahara, Chip control in turning with synchronization of spindle rotation and feed motion, Precision Engineering 53 (2018), 38-45

[2] P. Ostwald, Dynamic Chip Breaking - An Evaluation of the Effects Upon Surface Microgeometry and Free Chip Dimension, Oklahoma State University, 1966.

[3] R. Skeleton, Turning with an Oscillating Tool, International Journal of Machine Tool Design and Research 8 (1968), 239-259.

[4] M. Fuchs, C. Habersohn, Y. Kalkan, C. Lechner and F. Bleicher, Metrological Investigation of an Actuator Device for Vibration Assisted Turning, FME Transactions 43 (2015), 119-122.

[5] M. Nakamura, Y. Mitani, S. Asakura and T. Shibuya, The development of chip parting processing technology oscillatory feed cutting, JSAE Review 21 (2000), 555-559.

[6] J. Mann, Y. Guo, C. Saldana, W. Compton and S. Chandrasekar, Enhancing material removal processes using modulation-assisted machining, Tribology International 44 (2011), 1225-1235 